\title{
Multiplicity Dependence of Identical Particle Correlations in the Quantum Optical Approach
}

\author{
N. Suzuki and M. Biyajima* \\ Matsusho Gakuen Junior College, Matsumoto 390-1295, Japan \\ *Department of physics, Shinshu University, Matsumoto 390-8621, Japan
}

\begin{abstract}
Identical particle correlations at fixed multiplicity are considered in the presence of chaotic and coherent fields. The multiplicity distribution, one-particle momentum density and two-particle correlation function are obtained based on the diagrammatic representation for cumulants in semi-inclusive events. Our formulation is applied to the analysis of the experimental data on the multiplicity dependence of correlation functions reported by the UA1 and the OPAL Collaborations.
\end{abstract}

\section{Introduction}

In high energy hadron-hadron collisions, Bose-Einstein correlations of the identical particles are considered as one of the possible measures for the space-time domain where identical particles are produced. One of the theoretical approaches to the Bose-Einstein correlations is made on the analogy of the quantum optics [1], where two types of sources, chaotic and coherent are introduced. A diagrammatical method, based on the Glauber-Lachs formula [1], has been proposed [2] to find the higher order Bose-Einstein correlation (BEC) functions in the quantum optical (QO) approach. In Ref. [3], the generating functional (GF) for momentum densities is derived in the QO approach, and a diagrammatic representation for cumulants is proposed.

Up to the present, identical particle correlations in fixed multiplicity events are investigated in the case of purely chaotic field. Two-particle correlations are analyzed in Ref. [4] by using Monte Carlo methods. Multiplicity dependence of one-particle distributions is discussed in Ref. [5], and that of two or three-particle correlations are considered in Ref. [6].

In Ref. [7], an outline of our formulation on the particle correlations at fixed multiplicity in the QO approach has been briefly reported. General features of multiplicity distributions, one-particle distributions and two-particle correlations at fixed multiplicity have been also shown. In the present paper, identical particle correlations at fixed mutiplicity in the $\mathrm{QO}$ approach are considered in detail. The diagrammatic 
representation for cumulants is used to obtain the formulas in semi-inclusive events on the analogous way to that in inclusive events [3]. Furthermore, our formulas are applied to the analyses of the experimental data in $p \bar{p}$ collisions by the UA1 Collaboration [8], and in $e^{+} e^{-}$collisions by the OPAL Collaboration [9].

At first, we consider the case when there are no correlations among produced particles in the final states. Then particles in the final states are given by a coherent state,

$$
|\phi\rangle=\exp \left[-\frac{1}{2} \int|f(p)|^{2} \frac{d^{3} p}{E}+\int f(p) a^{\dagger}(p) \frac{d^{3} p}{E}\right]|0\rangle .
$$

The $n$-particle momentum density in semi-inclusive events is defined by

$$
\rho_{n}\left(p_{1}, \cdots, p_{n}\right)=\frac{1}{\sigma_{\text {inel }}} E_{1} \cdots E_{n} \frac{d^{3 n} \sigma_{\text {inel }}}{d^{3} p_{1} \cdots d^{3} p_{n}}=\left|\left\langle 0\left|a\left(p_{1}\right) \cdots a\left(p_{n}\right)\right| \phi\right\rangle\right|^{2},
$$

which is reduced to

$$
\rho_{n}\left(p_{1}, \cdots, p_{n}\right)=\left|f\left(p_{1}\right)\right|^{2} \cdots\left|f\left(p_{n}\right)\right|^{2} \exp \left[-\int|f(p)|^{2} \frac{d^{3} p}{E}\right] .
$$

In the QO approach, the function $f(p)$ is devided into two parts;

$$
f(p)=\sum_{i=1}^{M} a_{i} \phi_{i}(p)+f_{c}(p)
$$

where $\phi_{i}(p)$ and $f_{c}(p)$ are amplitudes of the $i$ th chaotic source and a coherent source, and $a_{i}$ is a random complex number attached to the $i$ th chaotic source. In addition, $M$ is the number of independent chaotic sources [3], which is regarded to be infinite in the present paper. The $n$-particle momentum density in the QO approach [10 is defined by,

$$
\rho_{n}\left(p_{1}, \cdots, p_{n}\right)=\left\langle\left|f\left(p_{1}\right)\right|^{2} \cdots\left|f\left(p_{n}\right)\right|^{2} \exp \left[-\int|f(p)|^{2} \frac{d^{3} p}{E}\right]\right\rangle_{a} .
$$

In Eq. (4), parenthesis $\langle F\rangle_{a}$ denotes an average of $F$ over the random number $a_{i}$ with a Gaussian weight [1];

$$
\langle F\rangle_{a}=\prod_{i=1}^{M}\left(\frac{1}{\pi \lambda_{i}} \int \exp \left[-\frac{\left|a_{i}\right|^{2}}{\lambda_{i}}\right] d^{2} a_{i}\right) F .
$$

It should be noticed that the classical (pion) fields are randomized in our approach. On the other hand, each mode of the light is randomized in the quantum optics [1]. After the average is taken over the random number $a_{i}$ in Eq. (5), terms composed of $a_{i}^{l} a_{i}^{* m}$ in the function $F$ vanish if $l \neq m$.

The generating functional (GF) for momentum densities in semi-inclusive events is defined by the following equation,

$$
Z_{\mathrm{sm}}[h(p)]=\sum_{n=1}^{\infty} \frac{1}{n !} \int \cdots \int \rho_{n}\left(p_{1}, \cdots, p_{n}\right) h\left(p_{1}\right) \cdots h\left(p_{n}\right) \frac{d^{3} p_{1}}{E_{1}} \cdots \frac{d^{3} p_{n}}{E_{n}}
$$


From Eqs. (4) and (6), the GF is written formally as,

$$
Z_{\mathrm{sm}}[h(p)]=\left\langle\exp \left[\int|f(p)|^{2}(h(p)-1) \frac{d^{3} p}{E}\right]\right\rangle_{a} .
$$

On the right hand side of Eq.(6), an additional constant $Z_{\mathrm{sm}}[h(p)=0]$, which does not affect to the momentum densities, is added. Inversely, the $n$-particle momentum density in the semi-inclusive events is given from the GF as

$$
\rho_{n}\left(p_{1}, \cdots, p_{n}\right)=\left.E_{1} \cdots E_{n} \frac{\delta^{n} Z_{\mathrm{sm}}[h(p)]}{\delta h\left(p_{1}\right) \cdots \delta h\left(p_{n}\right)}\right|_{h(p)=0} .
$$

From the sum rule between semi-inclusive and inclusive cross-sections [11], the GF $Z[h(p)]$ for inclusive events is connected to that for semi-inclusive events;

$$
Z[h(p)]=Z_{\mathrm{sm}}[h(p)+1]=\left\langle\exp \left[\int|f(p)|^{2} h(p) \frac{d^{3} p}{E}\right]\right\rangle_{a} .
$$

The $n$-particle inclusive momentum density is given by

$$
\rho_{\text {in }}\left(p_{1}, \cdots, p_{n}\right)=\left.E_{1} \cdots E_{n} \frac{\delta^{n} Z[h(p)]}{\delta h\left(p_{1}\right) \cdots \delta\left(p_{n}\right)}\right|_{h(p)=0} .
$$

The explicit form of the GF, Eq. (8), is shown in Ref. [3], and the higher order BEC functions in inclusive events are obtained from it. In the following section, we would like to show that we can obtain higher order momentum densities in semiinclusive events by analogy with a derivation in inclusive events.

\section{Generating functional and cumulant}

In the followings, we slightly change the definition of the GF from Eq.(7) to

$$
Z_{\mathrm{sm}}[h(p)]=c_{0}\left\langle\exp \left[\int|f(p)|^{2} h(p) \frac{d^{3} p}{E}\right]\right\rangle_{a},
$$

where the exponential damping factor in Eq. (7) is replaced by a normalization constant $c_{0}$. Then, the $n$-particle momentum density in the QO approach is given by

$$
\begin{aligned}
\rho_{n}\left(p_{1}, \cdots, p_{2}\right) & =\left.E_{1} \cdots E_{n} \frac{\delta^{n} Z[h(p)]}{\delta h\left(p_{1}\right) \cdots \delta\left(p_{n}\right)}\right|_{h(p)=0} \\
& =c_{0}\left\langle\left|f\left(p_{1}\right) \cdots f\left(p_{n}\right)\right|^{2}\right\rangle_{a}
\end{aligned}
$$

The GF $G_{\mathrm{sm}}[h(p)]$ for cumulants is defined by the following equation,

$$
G_{\mathrm{sm}}[h(p)] \equiv \ln Z_{\mathrm{sm}}[h(p)],
$$


and the $n$th order cumulant is given by

$$
g_{n}\left(p_{1}, \cdots, p_{n}\right)=\left.E_{1} \cdots E_{n} \frac{\delta^{n} G_{\mathrm{sm}}[h(p)]}{\delta h\left(p_{1}\right) \cdots \delta h\left(p_{n}\right)}\right|_{h(p)=0} .
$$

From Eqs. (10), (11) and (12), we have iteration relations for momentum densities,

$$
\begin{aligned}
\rho_{1}\left(p_{1}\right) & =c_{0} g_{1}\left(p_{1}\right) \\
\rho_{n}\left(p_{1}, \cdots, p_{n}\right) & =g_{1}\left(p_{1}\right) \rho_{n-1}\left(p_{2}, \cdots, p_{n}\right) \\
& +\sum_{i=1}^{n-2} \sum g_{i+1}\left(p_{1}, p_{j_{1}}, \cdots, p_{j_{i}}\right) \rho_{n-i-1}\left(p_{j_{i+1}}, \cdots, p_{j_{n-1}}\right) \\
& +c_{0} g_{n}\left(p_{1}, \cdots, p_{n}\right) .
\end{aligned}
$$

The second summation on the right hand side of Eq. (13) indicates that all possible combinations of $\left(j_{1}, \cdots, j_{i}\right)$ and $\left(j_{i+1}, \cdots, j_{n-1}\right)$ are taken from $(2,3, \cdots, n)$. Equation (13) shows that the $n$-particle momentum density $\rho_{n}\left(p_{1}, \cdots, p_{n}\right)(n=1,2, \cdots)$ can be evaluated if the cumulant $g_{n}\left(p_{1}, \cdots, p_{i}\right)(i=1,2, \cdots, n)$ is obtained.

The semi-inclusive one-particle and two-particle cumulants are given from Eq. (12) respectively as

$$
\begin{aligned}
g_{1}\left(p_{1}\right) & =\left\langle f\left(p_{1}\right)\right\rangle_{a}=r\left(p_{1}, p_{1}\right)+c\left(p_{1}, p_{1}\right) \\
g_{2}\left(p_{1}, p_{2}\right) & =\left\langle f\left(p_{1}\right) f\left(p_{2}\right)\right\rangle_{a}-\left\langle f\left(p_{1}\right)\right\rangle_{a}\left\langle f\left(p_{2}\right)\right\rangle_{a} \\
& =\left|r\left(p_{1}, p_{2}\right)\right|^{2}+2 \operatorname{Re}\left[r\left(p_{1}, p_{2}\right) c\left(p_{2}, p_{1}\right)\right]
\end{aligned}
$$

where $r\left(p_{1}, p_{2}\right)$ is a correlation caused by the chaotic sources and $c\left(p_{1}, p_{2}\right)$ is a correlation by the coherent source. Those are given by

$$
\begin{aligned}
r\left(p_{1}, p_{2}\right) & =\sum_{i=1}^{M} \lambda_{i} \phi_{i}\left(p_{1}\right) \phi_{i}^{*}\left(p_{2}\right), \\
c\left(p_{1}, p_{2}\right) & =f_{c}\left(p_{1}\right) f_{c}^{*}\left(p_{2}\right) .
\end{aligned}
$$

As the GF, Eq.(9), in semi-inclusive events is the same with the GF, Eq.(8), in inclusive events except for the normalization factor $c_{0}$, the cumulants of semiinclusive events are also calculated from the same diagrammatic presentation as those of inclusive events [3]. Diagrammatic representation for them up to the fourth order is shown in Fig.1.

The $n$th order cumulant is made up of connected terms of the correlations $r_{i j}$ of chaotic fields, and those $c_{i j}$ of the coherent field. If $n \geq 3$, the $n$th order cumulant $g_{n}\left(p_{1}, \cdots, p_{n}\right)$ is simply expressed by using the $n$-gon. The $n$th order cumulant consists of two types of terms; one is made only of the correlations of the chaotic fields, and the other contains the correlations both of the chaotic fields and of the coherent field. One of the terms belonging to the former type is given by $r_{12} r_{23} \cdots r_{(n-1) n} r_{n 1}$. It can be expressed by the circular permutation $(12 \cdots n)$ started from 1 . Other terms can be made from $(12 \cdots n)$ by any permutation. Therefore, there are $(n-1)$ ! different terms in the former type. If any one of the correlations $r_{i j}$ of the chaotic fields, 
belonging to the former type, is replaced by the correlation $c_{i j}$ of the coherent field, the terms of the latter type are made. Therefore, there are $n$ ! different terms in the latter type. It should be noted that the half of the terms in the $n$th order cumulant are complex conjugates to the other half.

\section{Basic formulas at fixed multiplicity}

In order to calculate momentum densities at fixed multiplicity, the $k$-particle momentum density and $k$ th order cumulant at $n$-particle events $(k \leq n)$ are defined by the following equations, respectively

$$
\begin{aligned}
& \rho_{n}^{(k)}\left(p_{1}, \cdots, p_{k}\right)=\frac{1}{(n-k) !} \int \cdots \int \rho_{n}\left(p_{1}, \cdots, p_{k}, p_{k+1}, \cdots, p_{n}\right) \frac{d^{3} p_{k+1}}{E_{k+1}} \cdots \frac{d^{3} p_{n}}{E_{n}} \\
& g_{n}^{(k)}\left(p_{1}, \cdots, p_{k}\right)=\frac{1}{(n-k) !} \int \cdots \int g_{n}\left(p_{1}, \cdots, p_{k}, p_{k+1}, \cdots, p_{n}\right) \frac{d^{3} p_{k+1}}{E_{k+1}} \cdots \frac{d^{3} p_{n}}{E_{n}} .
\end{aligned}
$$

The GF for multiplicity distribution $P(n)$ is given from Eq. (9) if function $h(p)$ is independent from momentum $p$;

$$
Z_{\mathrm{sm}}(h)=c_{0}\left\langle\exp \left[\int|f(p)|^{2} \frac{d^{3} p}{E} h\right]\right\rangle_{a} .
$$

The multiplicity distribution is given from Eq.(16);

$$
P(n)=\left.\frac{1}{n !} \frac{\partial^{n} Z_{\mathrm{sm}}(h)}{\partial h^{n}}\right|_{h=0}=\frac{1}{n !} \rho_{n}^{(0)} .
$$

The normalization of the $k$-particle momentum density at $n$-particle events is given by

$$
\int \cdots \int \rho_{n}^{(k)}\left(p_{1}, \cdots, p_{k}\right) \frac{d^{3} p_{1}}{E_{1}} \cdots \frac{d^{3} p_{k}}{E_{k}}=\frac{n !}{(n-k) !} P(n)
$$

Then the normalized $k$-particle momentum density in $n$-particle events is defined as

$$
\tilde{\rho}_{n}^{(k)}\left(p_{1}, \cdots, p_{k}\right)=\frac{(n-k) !}{n !} \frac{\rho_{n}^{(k)}\left(p_{1}, \cdots, p_{k}\right)}{P(n)} .
$$

In general, the inclusive $k$-particle momentum density is given from the semiinclusive momentum densities as

$$
\rho_{\text {in }}^{(k)}\left(p_{1}, \cdots, p_{k}\right)=\sum_{n=k} \rho_{n}^{(k)}\left(p_{1}, \cdots, p_{k}\right)
$$

which satisfies

$$
\int \cdots \int \rho_{\text {in }}^{(k)}\left(p_{1}, \cdots, p_{k}\right) \frac{d^{3} p_{1}}{E_{1}} \cdots \frac{d^{3} p_{k}}{E_{k}}=\sum_{n=k} \frac{n !}{(n-k) !} P(n) .
$$


It should be noted that if the $k$ th order inclusive momentum densities is integrated over all of the momenta, it becomes the $k$ th order factorial moment.

From Eqs. (13), (15) and (17), the multiplicity distribution, and particle densities in semi-inclusive events up to the third order can be expressed by the following recurrence equations,

$$
\begin{aligned}
P(n) & =\frac{1}{n} \sum_{j=1}^{n} j g_{j}^{(0)} P(n-j) \\
\rho_{n}^{(1)}\left(p_{1}\right) & =\sum_{j=1}^{n} g_{j}^{(1)}\left(p_{1}\right) P(n-j) \\
\rho_{n}^{(2)}\left(p_{1}, p_{2}\right) & =\sum_{j=1}^{n-1} g_{j}^{(1)}\left(p_{1}\right) \rho_{n-j}^{(1)}\left(p_{2}\right)+\sum_{j=2}^{n} g_{j}^{(2)}\left(p_{1}, p_{2}\right) P(n-j), \\
\rho_{n}^{(3)}\left(p_{1}, p_{2}, p_{3}\right) & =\sum_{j=1}^{n-2} g_{j}^{(1)}\left(p_{1}\right) \rho_{n-j}^{(2)}\left(p_{2}, p_{3}\right) \\
& +\sum_{j=2}^{n-1}\left\{g_{j}^{(2)}\left(p_{1}, p_{2}\right) \rho_{n-j}^{(1)}\left(p_{3}\right)+g_{j}^{(2)}\left(p_{1}, p_{3}\right) \rho_{n-j}^{(1)}\left(p_{2}\right)\right\} \\
& +\sum_{j=3}^{n} g_{j}^{(3)}\left(p_{1}, p_{2}, p_{3}\right) P(n-j)
\end{aligned}
$$

where $P(0)=c_{0}$. On the other hand, cumulants at fixed multiplicity are obtained from Fig.1 as

$$
\begin{aligned}
& g_{1}^{(0)}=\Delta_{1}^{(R)}+\Delta_{0}^{(S)} \\
& g_{n}^{(0)}=\frac{1}{n}\left[\Delta_{n}^{(R)}+2 \Delta_{n-1}^{(S)}+\sum_{j=1}^{n-2} \Delta_{j, n-j-1}^{(T)}\right], \quad n=2,3, \cdots \\
& g_{1}^{(1)}\left(p_{1}\right)= r\left(p_{1}, p_{1}\right)+c\left(p_{1}, p_{1}\right), \\
& g_{n}^{(1)}\left(p_{1}\right)= R_{n}\left(p_{1}, p_{1}\right)+2 S_{n-1}\left(p_{1}, p_{1}\right)+\sum_{j=1}^{n-2} T_{j, n-j-1}\left(p_{1}, p_{1}\right), \quad n=2,3, \cdots, \\
& g_{n}^{(2)}\left(p_{1}, p_{2}\right)= \sum_{j=1}^{n-1} R_{j}\left(p_{1}, p_{2}\right) R_{n-j}\left(p_{2}, p_{1}\right)+2 c\left(p_{1}, p_{2}\right) R_{n-1}\left(p_{2}, p_{1}\right) \\
&+ 2 \sum_{j=1}^{n-2}\left\{S_{j}\left(p_{1}, p_{2}\right) R_{n-j-1}\left(p_{2}, p_{1}\right)+R_{n-j-1}\left(p_{1}, p_{2}\right) S_{j}\left(p_{2}, p_{1}\right)\right\} \\
&+ \sum_{j=1}^{n-3} \sum_{l=1}^{n-j-2}\left\{T_{j, l}\left(p_{1}, p_{2}\right) R_{n-j-l-1}\left(p_{2}, p_{1}\right)+R_{n-j-l-1}\left(p_{1}, p_{2}\right) T_{j, l}\left(p_{2}, p_{1}\right)\right\} \\
& g_{n}^{(3)}\left(p_{1}, p_{2}, p_{3}\right)=\sum_{j=1}^{n-2} \sum_{l=1}^{n-j-1}\left\{R_{j}\left(p_{1}, p_{2}\right) R_{l}\left(p_{2}, p_{3}\right) R_{n-j-l}\left(p_{3}, p_{1}\right)\right.
\end{aligned}
$$




$$
\begin{aligned}
& \left.+R_{j}\left(p_{1}, p_{3}\right) R_{l}\left(p_{3}, p_{2}\right) R_{n-j-l}\left(p_{2}, p_{1}\right)\right\} \\
+ & 2 \sum_{j=1}^{n-2}\left\{c\left(p_{1}, p_{2}\right) R_{j}\left(p_{2}, p_{3}\right) R_{n-j-1}\left(p_{3}, p_{1}\right)\right. \\
& +c\left(p_{1}, p_{3}\right) R_{j}\left(p_{3}, p_{2}\right) R_{n-j-1}\left(p_{2}, p_{1}\right) \\
& \left.+c\left(p_{2}, p_{3}\right) R_{n-j-1}\left(p_{3}, p_{1}\right) R_{j}\left(p_{1}, p_{2}\right)\right\} \\
+ & \sum_{j=1}^{n-3} \sum_{l=1}^{n-j-2}\left\{S_{j}\left(p_{1}, p_{2}\right) R_{l}\left(p_{2}, p_{3}\right) R_{n-j-l-1}\left(p_{3}, p_{1}\right)\right. \\
& +S_{j}\left(p_{1}, p_{3}\right) R_{l}\left(p_{3}, p_{2}\right) R_{n-j-l-1}\left(p_{2}, p_{1}\right) \\
& +R_{j}\left(p_{1}, p_{2}\right) S_{l}\left(p_{2}, p_{3}\right) R_{n-j-l-1}\left(p_{3}, p_{1}\right) \\
& +R_{j}\left(p_{1}, p_{3}\right) S_{l}\left(p_{3}, p_{2}\right) R_{n-j-l-1}\left(p_{2}, p_{1}\right) \\
& +R_{j}\left(p_{1}, p_{2}\right) R_{n-j-l-1}\left(p_{2}, p_{3}\right) S_{l}\left(p_{3}, p_{1}\right) \\
& \left.+R_{j}\left(p_{1}, p_{3}\right) R_{n-j-l-1}\left(p_{3}, p_{2}\right) S_{l}\left(p_{2}, p_{1}\right)\right\} \\
& \quad \sum^{n-4} \sum_{j-j-3}^{n-j-l-2}\left\{\sum _ { m = 1 } \left\{T_{j, l}\left(p_{1}, p_{2}\right) R_{m}\left(p_{2}, p_{3}\right) R_{n-j-l-m-1}\left(p_{3}, p_{1}\right)\right.\right. \\
& j=1 \\
& +T_{j, l}\left(p_{1}, p_{3}\right) R_{m}\left(p_{3}, p_{2}\right) R_{n-j-l-m-1}\left(p_{2}, p_{1}\right) \\
& +R_{m}\left(p_{1}, p_{2}\right) T_{j, l}\left(p_{2}, p_{3}\right) R_{n-j-l-m-1}\left(p_{3}, p_{1}\right) \\
& +R_{m}\left(p_{1}, p_{3}\right) T_{j, l}\left(p_{3}, p_{2}\right) R_{n-j-l-m-1}\left(p_{2}, p_{1}\right) \\
& +R_{m}\left(p_{1}, p_{2}\right) R_{n-j-l-m-1}\left(p_{2}, p_{3}\right) T_{j, l}\left(p_{3}, p_{1}\right) \\
& \left.+R_{m}\left(p_{1}, p_{3}\right) R_{n-j-l-m-1}\left(p_{3}, p_{2}\right) T_{j, l}\left(p_{2}, p_{1}\right)\right\}, \\
&
\end{aligned}
$$

where

$$
\begin{aligned}
R_{1}\left(p_{1}, p_{2}\right)= & r\left(p_{1}, p_{2}\right) \\
R_{n}\left(p_{1}, p_{2}\right)= & \int r\left(p_{1}, k\right) R_{n-1}\left(k, p_{2}\right) \frac{d^{3} k}{\omega}, \quad n=2,3, \cdots, \\
S_{0}\left(p_{1}, p_{2}\right)= & c\left(p_{1}, p_{2}\right), \\
S_{n}\left(p_{1}, p_{2}\right)= & \int c\left(p_{1}, k\right) R_{n}\left(k, p_{2}\right) \frac{d^{3} k}{\omega}, \quad n=1,2, \cdots, \\
T_{j, l}\left(p_{1}, p_{2}\right)= & \iint R_{j}\left(p_{1}, k_{1}\right) c\left(k_{1}, k_{2}\right) R_{l}\left(k_{2}, p_{2}\right) \frac{d^{3} k_{1}}{\omega_{1}} \frac{d^{3} k_{2}}{\omega_{2}} \\
& \Delta_{n}^{(R)}=\int R_{n}(k, k) \frac{d^{3} k}{\omega} \\
& \Delta_{n}^{(S)}=\int S_{n}(k, k) \frac{d^{3} k}{\omega} \\
& \Delta_{j, l}^{(T)}=\int T_{j, l}(k, k) \frac{d^{3} k}{\omega} .
\end{aligned}
$$

In the followings, variables are changed from $\left(p_{1 L}, \mathbf{p}_{1 T}\right)$ to $\left(y_{1}, \mathbf{p}_{1 T}\right)$, with rapidity $y_{1}=\tanh ^{-1}\left(p_{1 L} / E_{1}\right)$. Both correlations $r\left(p_{1}, p_{2}\right)$ and $c\left(p_{1}, p_{2}\right)$ are assumed to be real 
and parametrized as,

$$
\begin{aligned}
r\left(y_{1}, \mathbf{p}_{1 T} ; y_{2}, \mathbf{p}_{2 T}\right) & =p \sqrt{\rho\left(y_{1}, \mathbf{p}_{1 T}\right) \rho\left(y_{2}, \mathbf{p}_{2 T}\right)} I\left(\Delta y, \Delta \mathbf{p}_{1 T}\right), \\
c\left(y_{1}, \mathbf{p}_{1 T} ; y_{2}, \mathbf{p}_{2 T}\right) & =(1-p) \sqrt{\rho\left(y_{1}, \mathbf{p}_{1 T}\right) \rho\left(y_{2}, \mathbf{p}_{2 T}\right)}, \\
\rho\left(y_{1}, \mathbf{p}_{1 T}\right) & =<n_{0}>\sqrt{\frac{\pi}{\alpha}} \frac{\pi}{\beta} \exp \left[-\alpha y_{1}^{2}-\beta \mathbf{p}_{1 T}^{2}\right], \\
I\left(\Delta y, \Delta \mathbf{p}_{T}\right) & =\exp \left[-\gamma_{L}(\Delta y)^{2}-\gamma_{T}\left(\Delta \mathbf{p}_{T}\right)^{2}\right],
\end{aligned}
$$

where $p=r\left(p_{i}, p_{i}\right) / \rho\left(p_{i}\right)$ is called the chaoticity parameter, $\Delta y=y_{2}-y_{1}$ and $\Delta \mathbf{p}_{T}=$ $\mathbf{p}_{2 T}-\mathbf{p}_{1 T}$. Functions defined by Eqs. (27), (28) and (29) are expressed as

$$
\begin{aligned}
& R_{j}\left(y_{1}, \mathbf{p}_{1 T}, y_{2}, \mathbf{p}_{2 T}\right)=N_{j} \exp \left[-A_{j}\left(y_{1}^{2}+y_{2}^{2}\right)+2 C_{j} y_{1} y_{2}\right] \\
& \times \exp \left[-U_{j}\left(\mathbf{p}_{1 T}^{2}+\mathbf{p}_{2 T}^{2}\right)+2 W_{j} \mathbf{p}_{1 T} \cdot \mathbf{p}_{2 T}\right], \\
& S_{j}\left(y_{1}, \mathbf{p}_{1 T}, y_{2}, \mathbf{p}_{2 T}\right)= \frac{(1-p)<n_{0}>\alpha^{1 / 2} \beta}{\sqrt{A_{j}+\alpha / 2}\left(U_{j}+\beta / 2\right)} N_{j} \\
& \times \exp \left[-\frac{\alpha}{2} y_{1}^{2}-\left(\frac{\alpha}{2}+\frac{\alpha \gamma_{L}}{A_{j}+\alpha / 2}\right) y_{2}^{2}\right], \\
& T_{i, j}\left(y_{1}, \mathbf{p}_{1 T}, y_{2}, \mathbf{p}_{2 T}\right)=\frac{\exp \left[-\frac{\beta}{2} \mathbf{p}_{1 T}^{2}-\left(\frac{\beta}{2}+\frac{\beta \gamma_{T}}{A_{j}+\beta / 2}\right) \mathbf{p}_{2 T}^{2}\right],}{\sqrt{\left(A_{i}+\alpha / 2\right)\left(A_{j}+\alpha / 2\right)}\left(U_{i}+\beta / 2\right)\left(U_{j}+\beta / 2\right)} N_{i} N_{j} \\
& \times \exp \left[-\left(\frac{\alpha}{2}+\frac{\alpha \gamma_{L}}{A_{i}+\alpha / 2}\right) y_{1}^{2}-\left(\frac{\alpha}{2}+\frac{\alpha \gamma_{L}}{A_{j}+\alpha / 2}\right) y_{2}^{2}\right] \\
& \times \exp \left[-\left(\frac{\beta}{2}+\frac{\beta \gamma_{T}}{A_{i}+\beta / 2}\right) \mathbf{p}_{1 T}^{2}-\left(\frac{\beta}{2}+\frac{\beta \gamma_{T}}{A_{j}+\beta / 2}\right) \mathbf{p}_{2 T}^{2}\right],
\end{aligned}
$$

where

$$
\begin{aligned}
A_{1} & =\frac{\alpha}{2}+\gamma_{L}, \quad C_{1}=\gamma_{L}, \\
A_{j+1} & =A_{1}-\frac{\gamma_{L}^{2}}{A_{j}+A_{1}}, \quad C_{j+1}=\frac{\gamma_{L} C_{j}}{A_{j}+A_{1}}, \\
U_{1} & =\frac{\beta}{2}+\gamma_{T}, \quad W_{1}=\gamma_{T}, \\
U_{j+1} & =U_{1}-\frac{\gamma_{T}^{2}}{U_{j}+U_{1}}, \quad W_{j+1}=\frac{\gamma_{T} W_{j}}{U_{j}+U_{1}}, \\
N_{1} & =p<n_{0}>\frac{\alpha^{1 / 2} \beta}{\pi^{3 / 2}}, \\
N_{j+1} & =\frac{p<n_{0}>\alpha^{1 / 2} \beta}{\sqrt{A_{j}+A_{1}}\left(U_{j}+U_{1}\right)} N_{j} .
\end{aligned}
$$




\section{Analyses of experimental data}

Our formulas are applied to the analyses of negatively charged particles, or likesign particles. Recently, the preliminary data of identical two-particle correlations in semi-inclusive events in $p \bar{p}$ collisions at $\sqrt{s}=900 \mathrm{GeV}$ within the pseudo-rapidity interval from -3.0 to 3.0 are reported by the UA1 Collaboration [8]. As can be seen from Eq. (21), multiplicity distribution and one-particle densities are included in the formula of the two-particle density, at least the multiplicity distribution of the same data sample is required to analyze the two-particle correlation. The multiplicity distribution at $\sqrt{s}=900 \mathrm{GeV}$ is also reported by the UA1 group [12]. However, the data are taken within the pseudo-rapidity interval from -2.5 to 2.5 . In the present analysis, those data are used to adjust the parameters included in our formulation.

Parameter $\alpha$ is determined from the parametrization of one-particle rapidity distribution of Landau's hydrodynamical model [13], and $\beta$ is taken from the inclusive transverse momentum distribution. In the present analysis, we neglect the correlation in the transverse momentum space, in other words, $\gamma_{T}$ is taken to be zero, and the calculated value is compared with the data after integrated over the transverse momentum. Therefore, the parametrization of $\beta$ does not affect the calculated results. Those values are taken as

$$
\alpha=0.25, \quad \beta=5.556, \quad \gamma_{T}=0 .
$$

Multiplicity distribution is normalized to satisfy

$$
\sum_{n=1}^{n_{\max }} P(n)=1
$$

where $n_{\max }=42$ is the maximum multiplicity of the observed negatively charged particles.

Other parameters are adjusted to fit the multiplicity distribution [12 from $n=1$ to $n=35$ in the following way. The chaoticity parameter $p$ is changed from $p=0$ to $p=1.0$ by the step 0.1 , and other parameters $\left\langle n_{0}\right\rangle$ and $\gamma_{L}$ are determined by the minimum chi-squared method. The best fit is given by

$$
p=0.8, \quad\left\langle n_{0}\right\rangle=2.584, \quad \gamma_{L}=1.394,
$$

with $\chi_{\min }^{2} / \mathrm{NDF}=372.4 / 32$. As can be seen from Eq. (29), if we keep the relations that $\alpha / \gamma_{L}=$ costant and $\beta / \gamma_{T}=$ constant, we get the same minimum chi-squared value. The calculated multiplicity distribution is compared with the data in Fig.2. For the sake of comparison, we also fit the data by the negative binomial distribution, which results in $\chi_{\min }^{2} / \mathrm{NDF}=465.0 / 33$.

The normalized one-particle rapidity distribution at $n$-particle events is defined by

$$
\tilde{\rho}_{n}^{(1)}(y)=\int \tilde{\rho}_{n}^{(1)}\left(y, \mathbf{p}_{T}\right) d^{2} \mathbf{p}_{T}
$$

and calculated results for $n=5,10$ and 20 are shown in Fig.3. The peak height increases and the width of the distribution becomes narrower, as the multiplicity $n$ increases. 
In Fig.4, the normalized two-particle rapidity distribution given by

$$
\tilde{\rho}_{n}^{(2)}(\Delta y)=\iiint \tilde{\rho}_{n}^{(2)}\left(y_{1}, \mathbf{p}_{1 T}, y_{1}+\Delta y, \mathbf{p}_{2 T}\right) d y_{1} d^{2} \mathbf{p}_{1 T} d^{2} \mathbf{p}_{2 T}
$$

are shown at $n=5,10$ and 20 . The peak of the distribution also becomes higher and it's width becomes narrower, as the multiplicity $n$ increases. However the increasing rate is gentler than that of the one-particle density.

The normalized two-particle correlation function $C_{n}^{(2-)}(\Delta y)$ at $n$-particle events is defined as

$$
C_{n}^{(2-)}(\Delta y)=\frac{\iiint \tilde{\rho}_{n}^{(2)}\left(y_{1}, \mathbf{p}_{1 T}, y_{1}+\Delta y, \mathbf{p}_{2 T}\right) d y_{1} d^{2} \mathbf{p}_{1 T} d^{2} \mathbf{p}_{2 T}}{\iiint \tilde{\rho}_{n}^{(1)}\left(y_{1}, \mathbf{p}_{1 T}\right) \tilde{\rho}_{n}^{(1)}\left(y_{1}+\Delta y, \mathbf{p}_{2 T}\right) d y_{1} d^{2} \mathbf{p}_{1 T} d^{2} \mathbf{p}_{2 T}}-1
$$

The calculated result on $C_{n}^{(2-)}(\Delta y)$ at $n=5,10$ and 20 are shown on Fig.5. The multiplicity dependence of $C_{n}^{(2-)}(\Delta y)$ at $\Delta y=0$ and 1.5 are shown in Fig.6, where the preliminary experimental data reported by the UA1 Collaboration at $Q=0.1 \mathrm{GeV}$ are also shown $\rrbracket$.

In $e^{+} e^{-}$collisions, the OPAL Collaboration published the data on multiplicity distributions [14], and multiplicity dependence of two-particle Bose-Einstein correlations [9] at $91 \mathrm{GeV}$. However, using the parameters adjusted to the observed multiplicity distribution, which is close to a Poisson distribution, calculated results on $C_{n}^{(2-)}(\Delta y=0)$ are almost constant and does not show the $n$ dependence. Next, the multiplicity dependence of the Bose-Einstein correlations at $Q=0 \mathrm{GeV}$, which is estimated from the data with $Q \geq 0.05 \mathrm{GeV}$, is directly analyzed by our formula. We can fairly well reproduce the $n$ dependence of the data with $n_{\max }=27, \alpha=0.125$, $p=0.55$ and $\gamma_{L}=10.0$, if the minimum values of our calculated results on $C_{n}^{(2-)}(0)+1$ are renormalized to 1. The result is shown in Fig.7.

\section{$5 \quad$ Summary and discussions}

The analytical formulas of multiplicity distribution and particle densities in semiinclusive events are derived from the generating functional GF in the presence of the chaotic and coherent fields. Formulas are applied to the analysis of the multiplicity dependence of two-particle correlations among identical particles in $p \bar{p}$ Collisions by the UA1 Collaboration [8] and in $e^{+} e^{-}$collisions by the OPAL Collaboration [9]. In the formula of two-particle correlation, multiplicity distribution and one-particle densities in semi-inclusive events are contained. Therefore, to fix the parameters in our formulas, at least the observed multiplicity distribution is necessary.

In $p \bar{p}$ collisions, we adjusted the parameters using the multiplicity distribution taken from the different data sample from those of the two-particle correlation. Our calculated results with the constant chaoticity parameter well reproduce the gross

\footnotetext{
${ }^{1}$ The data on the two-particle correlation are given by the variable 4- momentum transfer squared; $Q=\sqrt{-\left(p_{1}-p_{2}\right)^{2}} \mathrm{GeV} . Q=0$ corresponds to $\Delta y=0$. Therefore we compare our calculated results at $\Delta y=0$ with the data at the smallest $Q$ value $(Q=0.1)$ reported by the UA1 Collaboration.
} 
features of the multiplicity dependence of the data, inspite of the values of $C_{n}^{(2)}(0)$ are smaller than the data at $Q=0.1 \mathrm{GeV}$ about $20 \%$. Some part of the deviation will be deduced to the fact that the parameters are determined by fitting the multiplicity distribution within a different pseudo-rapidity interval.

In $e^{+} e^{-}$collisions, we analyze the data of the two-particle correlation without fitting the multiplicity distribution. We can explain the multiplicity dependence of the two-particle correlation at $Q=Q_{\text {min }} \mathrm{GeV}$ observed in the experiment, using the constant chaoticity parameter.

Calculated results on the normalized two-particle correlation in semi-inclusive events show that the peak of the distribution becomes lower as the multiplicity increases, even if the chaoticity parameter $p$ is constant. This behavior is similar to the data of two-particle correlations in $p \bar{p}$ collisions by the UA1 Collaboration, and in $e^{+} e^{-}$collisions by the OPAL Collaboration.

In this paper, we analyze the data with the same values of chaoticity parameter $p$ and the correlation length $\gamma_{L}$ in rapidity space. Our present analyses indicate that the coherent component is not negligible, in other words, the values of chaoticity parameters are smaller than 1 . One of the possible candidates for the coherent component is a contribution from the decay products of long lived resonances [15]. Another possibility to reduce the value of chaoticity parameter is contanination [16, 3]. For example, about $20 \%$ of like-sign particles are not pions in the OPAL Collaboration [9].

When the colliding energy of incident particles increases as in the forthcoming RHIC experiment, thousands of identical particles can be produced in an event. Then, the production domain of those particles can be analysed precisely event by event. In general, the values of parameters will change according to the multiplicity. If the fitted values of chaoticity parameter or correlation length change suddenly at some multiplicity, it will be a possible signature that a threshold of a new phenomenon will open at that multiplicity.

Acknowledgments: M.B. is partially supported by the Japanese Grant-inAid for Scientific Research from the Ministry of Education, Science and Culture (No.09440103) and (No.08304024). N.S. thanks for A.Bartl, B.Buschbeck and H.Eggers for valuable discussions. N.S. also thanks Matsusho Gakuen Junior College for financial support.

\section{References}

[1] R. J. Glauber, Phys. Rev. 131, 2766(1963); in Proceedings of the Les Houches Summer School of Theoretical Physics, Quantum Optics and Electronics, Grenoble, 1964 edited by C. De Witt, A. Blandin and C. Cohen-Tannoudji (Gordon and Breach, New York, 1965), p.63; G. Lachs, Phys. Rev., 138, B1012(1965).

[2] M.Biyajima, A.Bartl, T.Mizoguchi, N.Suzuki and O.Terazawa, Prog. Theor. Phys. 84, 931(1990); ibid. 88, 157(1992). 
[3] N.Suzuki, M.Biyajima and I.V.Andreev, Phys. Rev. C56, 2736(1997); N.Suzuki and M. Biyajima, Prog. Theor. Phy. 88, 609(1992).

[4] W. A. Zajc, Phys. Rev. D35, 3396(1987).

[5] S. Pratt, Phys. Lett. B301, 159(1993).

[6] W.Q.Chao, C.S.Gao and Q.H.Zhang, J. Phys. G21, 847(1995); Q.H.Zhang, Phys. Lett., B406, 365(1997).

[7] N.Suzuki and M.Biyajima, in Proceedings of the 8th International Symposium on Multiparticle Production "Correlation and Fluctuations '98", Matrahaza, Hungary,1998, edited by T.Csorgo, S. Hegyi, R.C.Hwa and G.Jancso, (World Scientific, Singapore, 1999), p.98

[8] B.Buschbeck, private communication; B.Buschbeck, H.Eggers and P.Lipa, HEPHY-PUB 700/98, in Proceedings of the 8th International Symposium on Multiparticle Production "Correlation and Fluctuations '98", Matrahaza, Hungary, 1998, edited by T.Csorgo, S. Hegyi, R.C.Hwa and G.Jancso, (World Scientific, Singapore, 1999), p.28

[9] OPAL Collaboration, G.Alexander et al., Z. Phys. C72, 389(1996)

[10] M. Biyajima, O. Miyamura and T. Nakai, in Proceedings of the Multiparticle Dynamics, Hakone, Japan,1978, edited by T. Hirose et al. (PIFP, Kyoto Univ., Japan, 1978), p.139.

[11] Z. Koba, H. B. Nielsen and P. Olesen, Nulcl. Phys. B43, 125(1972); Z.Koba, Acta Phys. Pol. B4, 95(1973). L. S. Brown, Phys. Rev. D5, 748(1972).

[12] UA1 Collaboration, C.Albajar et al., Nucl. Phys. B335, 1261(1990)

[13] P. Carruthers and Minh Duong-van, Phys. Rev. D8, 859(1973)

[14] OPAL Collaboration, P.D.Acton et al., Z. Phys. C52, 539(1992)

[15] M. G. Bowl, Particle World 2, 1(1991)

[16] J. G. Cramer and K. Kadija, Phys. Rev. C53, 908(1996)

\section{Figure Captions}

Fig.1 Diagrammatic representation for cumulants in semi-inclusive events.

a) Contribution from the chaotic field, $r\left(p_{1}, p_{2}\right)$, is shown by the solid line with arrow orienting from point 1 to 2 . That of the coherent field, $c\left(p_{1}, p_{2}\right)$, is shown by dotted line with arrow from 1 to 2 .

b) Diagram for $g_{2}\left(p_{1}, p_{2}\right)$.

c) Diagram for $g_{3}\left(p_{1}, p_{2}, p_{3}\right)$. All permutations of $(2,3)$ should be taken for $(i, j)$.

d) Diagram for $g_{4}\left(p_{1}, \cdots, p_{4}\right)$. Those of $(2,3,4)$ should be taken for $(i, j, k)$ 
Fig.2 Multiplicity distribution observed in $p \bar{p}$ collisions [14 is analyzed by our formula. Parameters are determined by the minimum chi-squared method: $p=0.8,<n_{0}>=2.584$ and $\gamma_{L}=1.394$.

Fig.3 Normalized one-particle rapidity distributions at fixed multiplicity calculated with $p=0.8,<n_{0}>=2.584$ and $\gamma_{L}=1.394$.

Fig.4 Normalized two-particle rapidity distributions at fixed multiplicity calculated with $p=0.8,<n_{0}>=2.584$ and $\gamma_{L}=1.394$.

Fig.5 Normalized two-particle correlation functions at fixed multiplicity calculated with $p=0.8$ and $\gamma_{L}=1.394$.

Fig.6 Multiplicity dependence of normalized two-particle correlations. Full circles show the data at $Q=0.1 \mathrm{GeV}$ in $p \bar{p}$ collisions 8 . Open circles and open squares are calculated with $p=0.8$ and $\gamma_{L}=1.394$.

Fig.7 Multiplicity dependence of normalized two-particle correlations. Full circles indicate the values at $Q=0 \mathrm{GeV}$, estimated from the data with $Q \geq 0.05 \mathrm{GeV}$ in $e^{+} e^{-}$collisions [9]. Open circles are obtained from our calculation at $\Delta y=0$. 


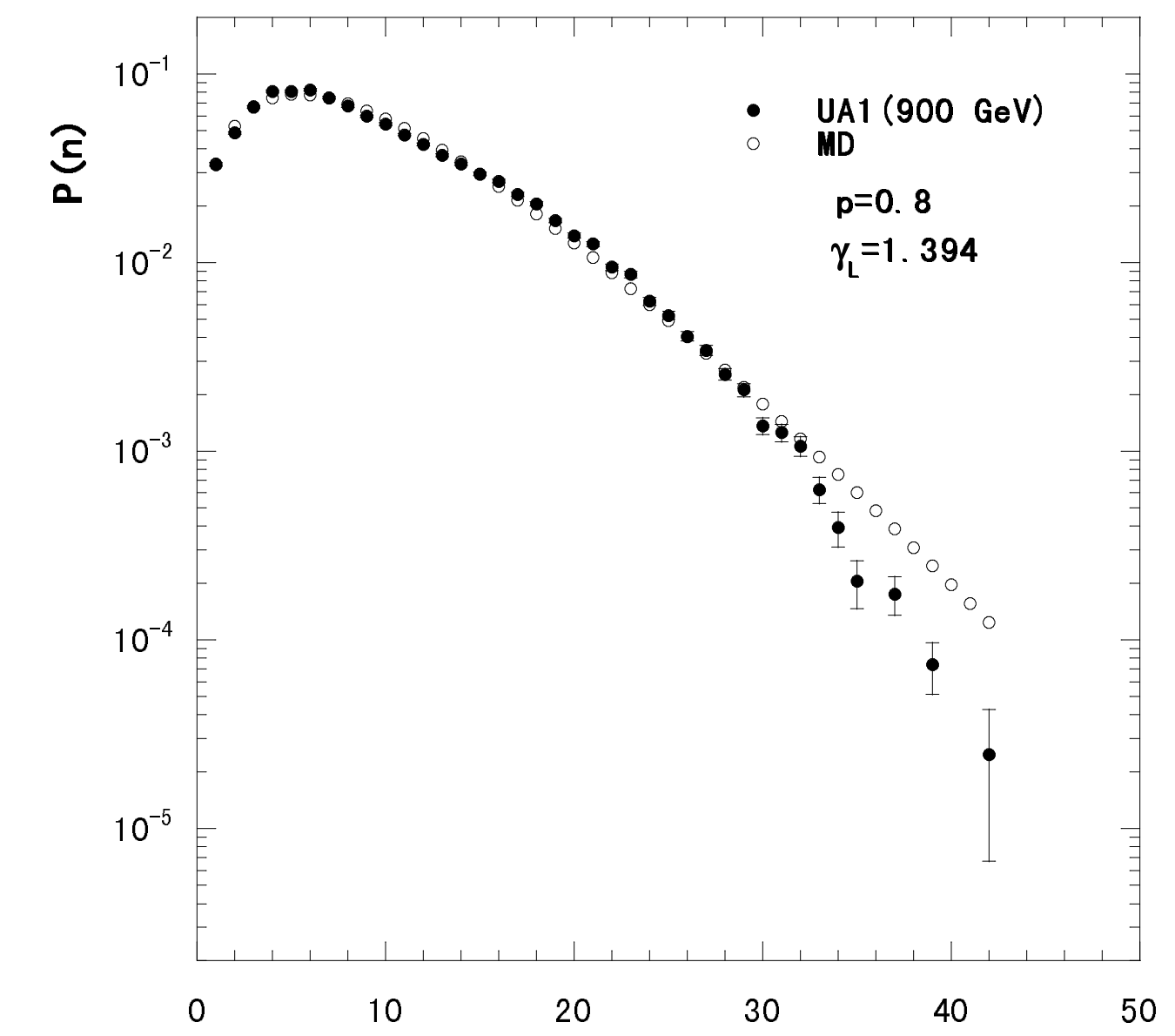




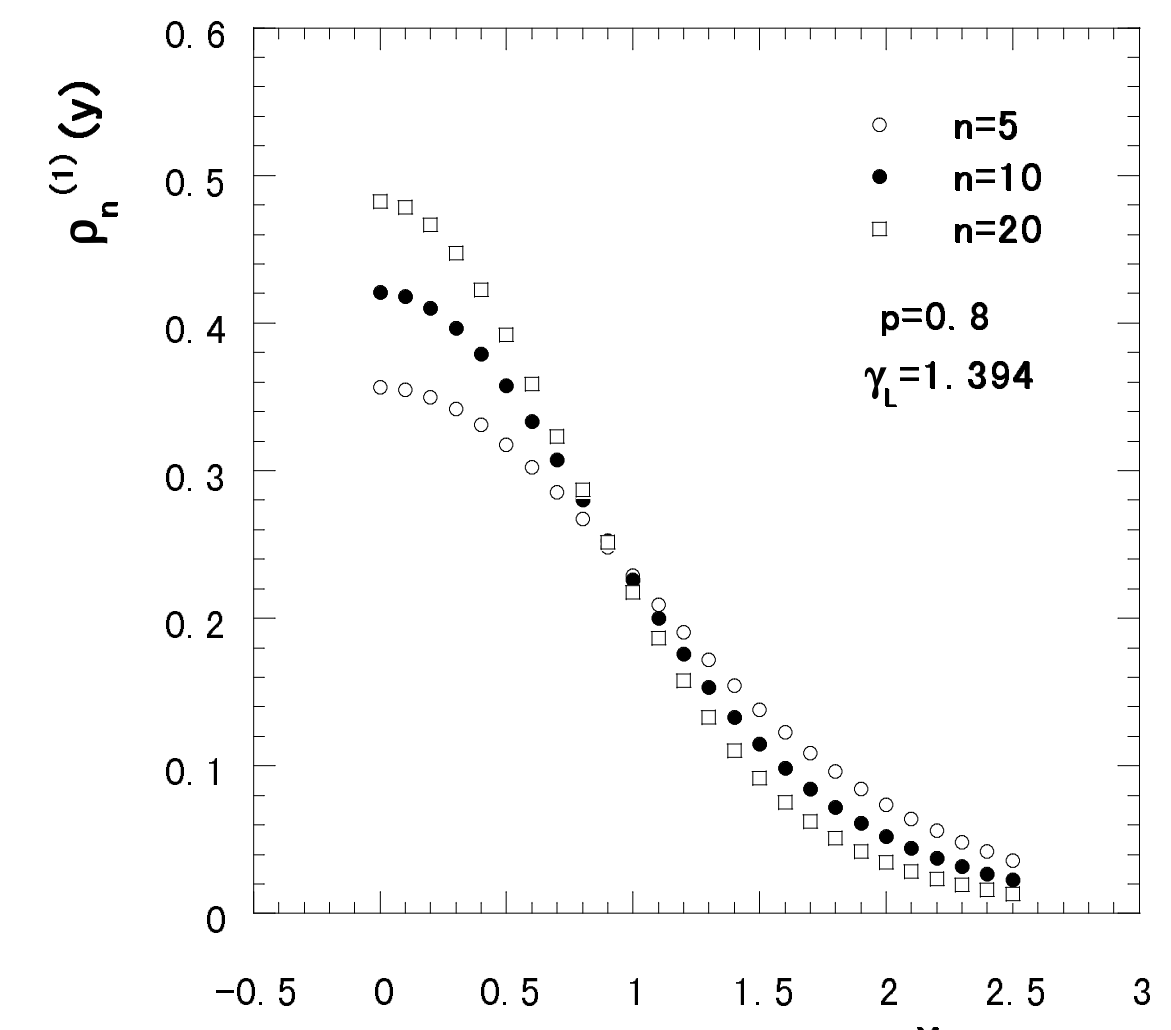




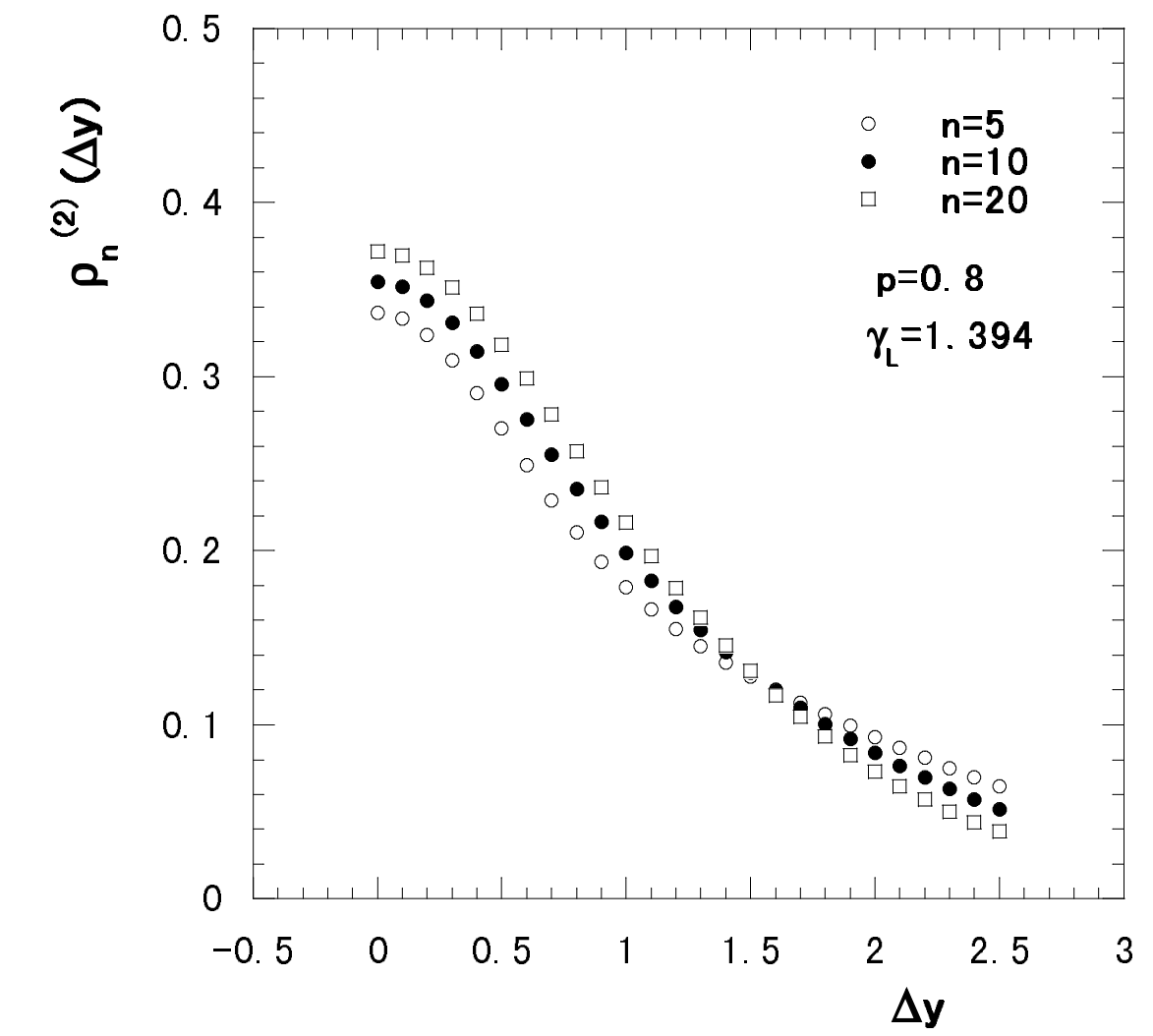




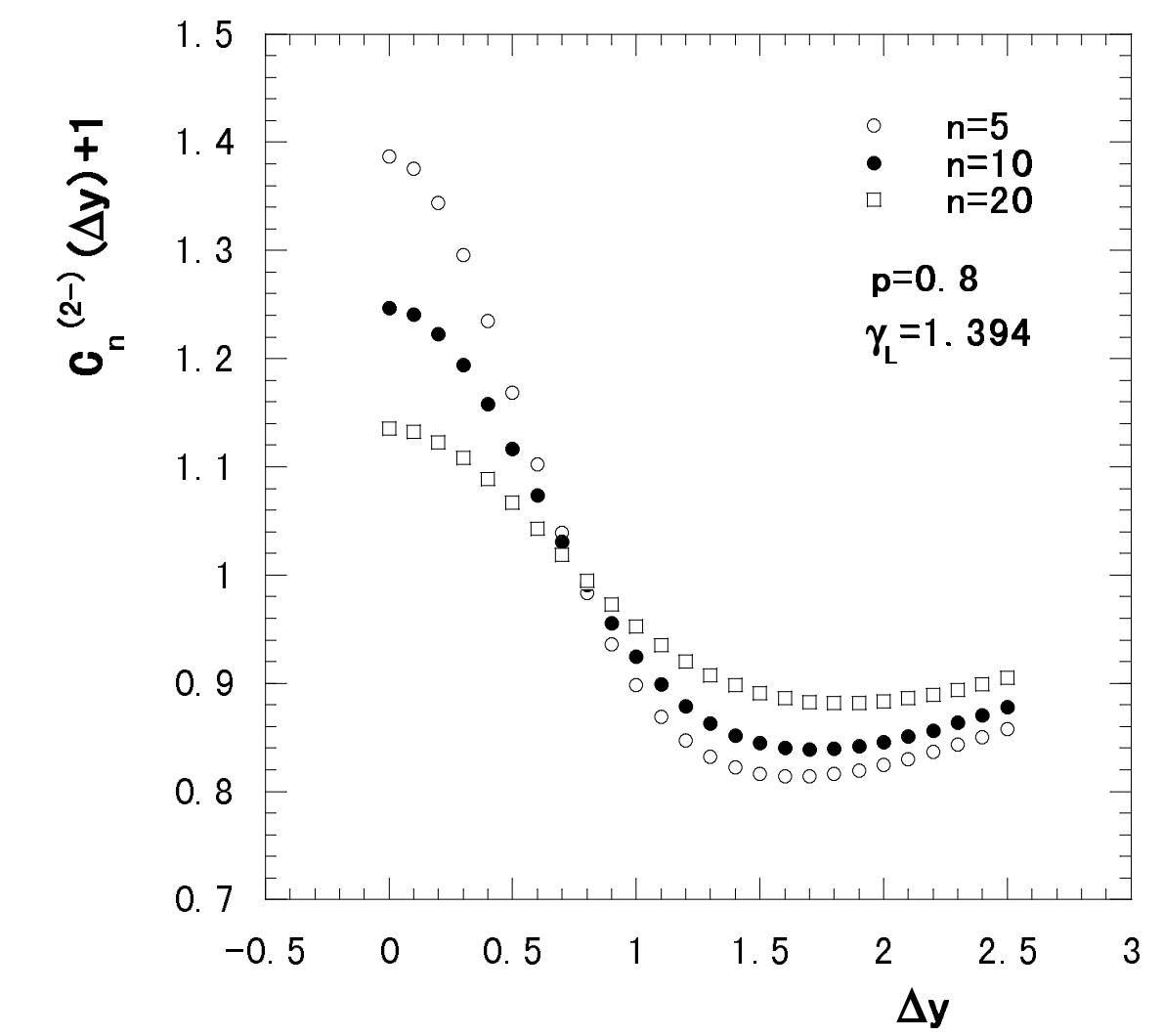




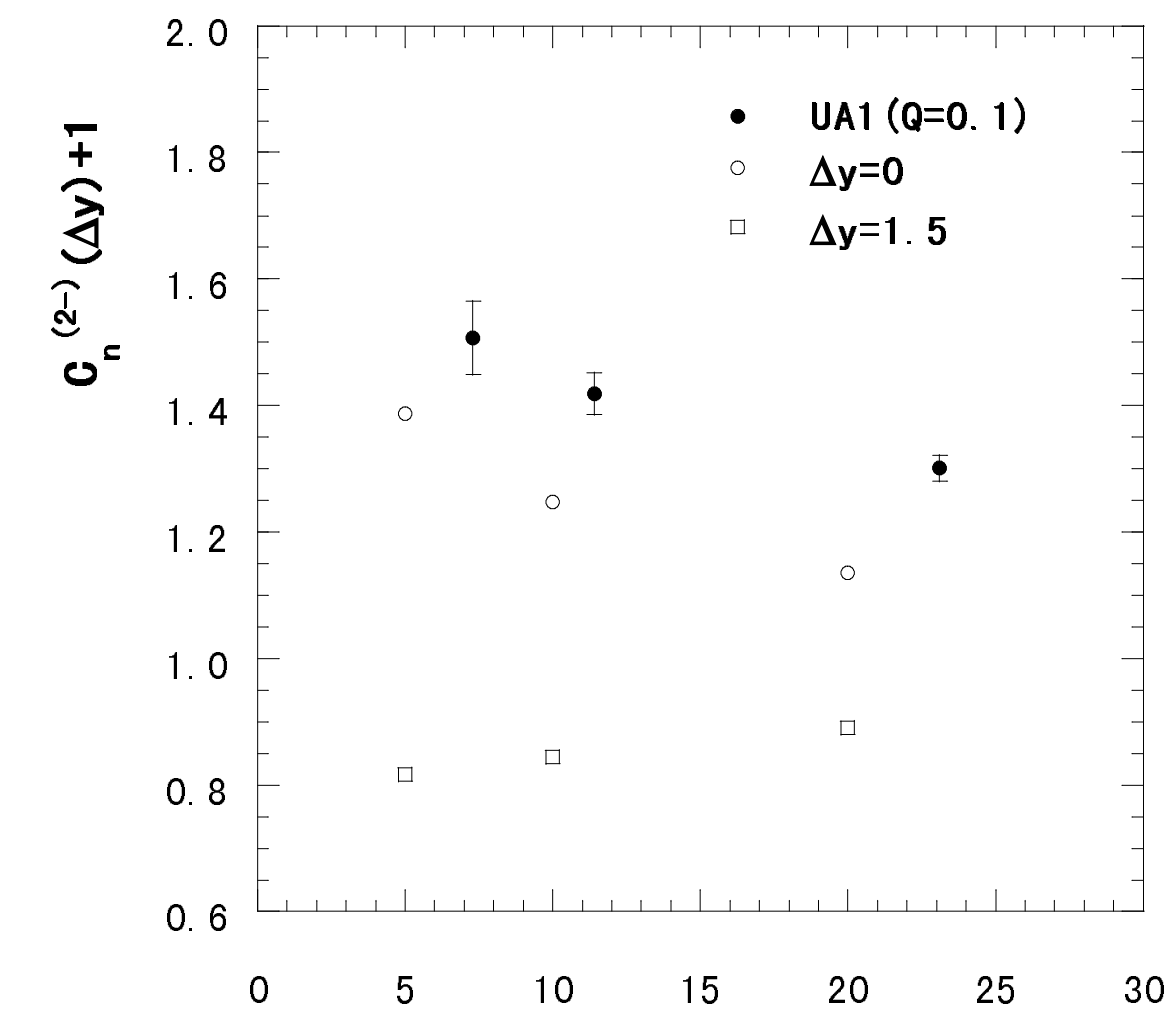




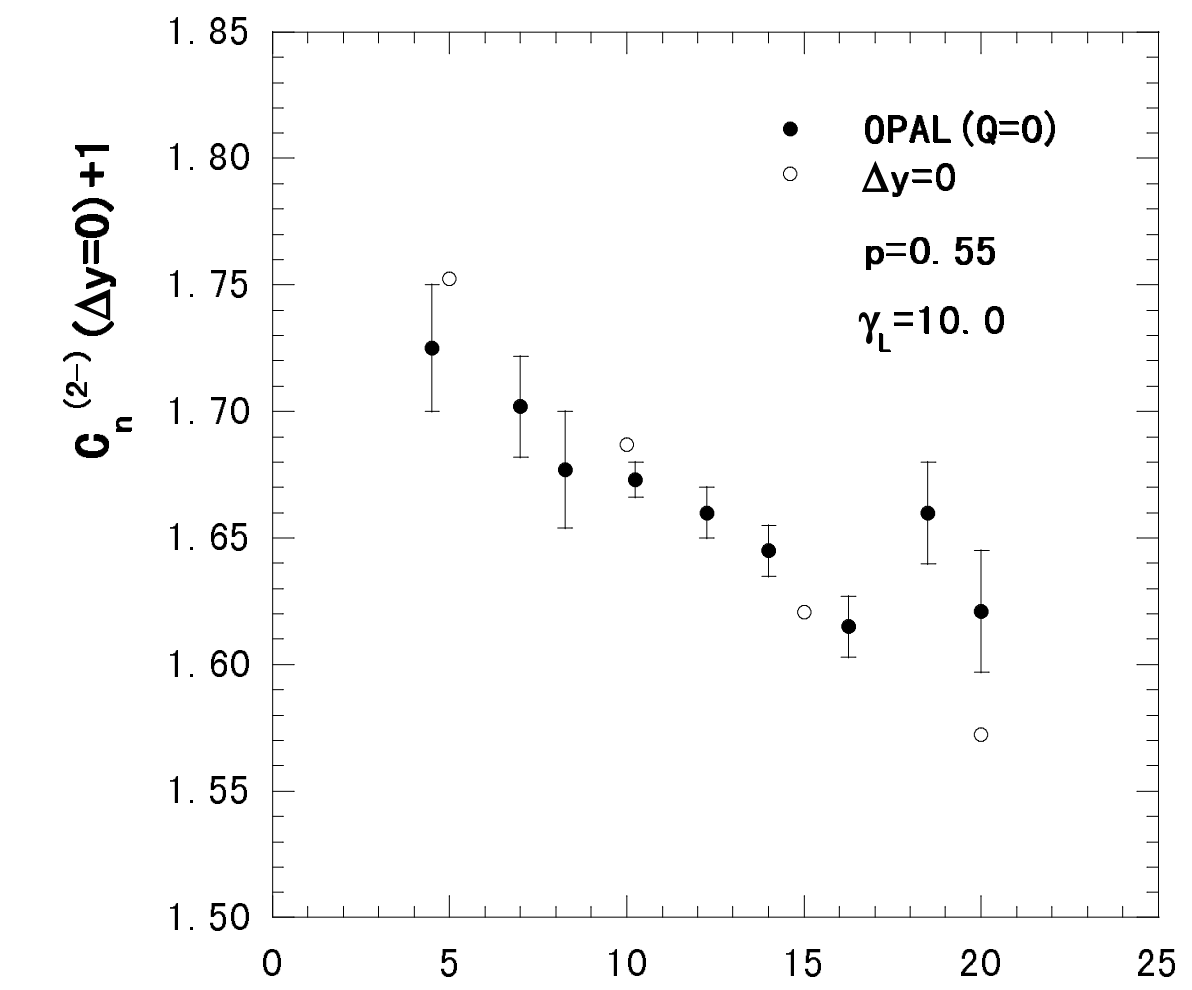

